\title{
Noncoherent Detection Based on Markov Chain Monte Carlo Methods for Block Fading Channels
}

\author{
Rong-Rong Chen and Ronghui Peng \\ Department of Electrical and Computer Engineering, University of Utah, UT 84112 \\ Email: \{rchen, peng\}@ece.utah.edu
}

\begin{abstract}
In this work we study joint channel decoding and noncoherent detection for block fading channels. We propose a novel, low-complexity noncoherent detection method based on Markov Chain Monte Carlo (MCMC). The MCMC noncoherent detector makes it possible to use large constellations such as $\mathbf{1 6}$ QAM and transmit at higher rates of 1 or 1.6 bits/channel use. By employing joint channel decoding and noncoherent detection, the proposed schemes achieve within 1.2-1.4 dB of the noncoherent channel capacity. Moreover, for the same transmission rates, the proposed single transmit antenna system performs 4-6 dB better than published results of the two transmit antenna systems that employ unitary space-time codes or orthogonal space-time codes.
\end{abstract}

\section{INTRODUCTION}

In this paper, we investigate the design of joint channel decoding and data detection/demodulation schemes for block fading channels. We focus on the noncoherent scenario where neither the transmitter nor the receiver has perfect channel state information. In order to approach channel capacity, we consider systems where a powerful channel code is concatenated with a modulation code to strengthen the error-correcting capability. Iterative receivers are employed to allow soft information exchange between the decoder and the detector.

In recent work ([1]-[3]), aiming to exploit the capacity gain promised by multiple-input and multiple-output (MIMO) channels, performances of channel codes in conjunction with modulation codes such as unitary space-time codes are investigated. Motivations for using unitary space-time codes come from the fact that isotropically distributed unitary signals achieve the capacity of block fading channels when the coherence length is much larger than the number of transmit antenna or at high SNR [4]. However, a disadvantage for using unitary signals lies in the difficulty of developing low-complexity detectors that can efficiently separate these unitary matrices. Typically, as in [1]-[3], only a small set (up to 1024) of unitary matrices are used. This may potentially incur a transmission rate loss compared to the optimal capacity achieved by the infinite set of isotropically distributed unitary signals.

In this paper, we develop novel, low-complexity noncoherent detectors based on the Markov Chain Monte Carlo (MCMC) approach. The proposed detectors are capable of decoding large size modulation codes and achieving high transmission rates. Applications of the MCMC detectors for coherent MIMO channels (where the receiver has perfect channel state information) have been studied in [5]-[8], which show great performance improvement over traditional MIMO detectors such as the sphere decoding detectors. In this work, we extend the application of the MCMC approach to the noncoherent scenario and show that our coded systems with single transmit and receive antenna can achieve within 1.2$1.4 \mathrm{~dB}$ of the channel capacity for the transmission rates of 1 and 1.6 bits/channel use. These results have a gain of 4-6 dB compared to the two transmit antenna / one receive antenna systems in ([1]-[3]).

To explore the possibility of improving the modulation codes in ([1]-[3]) for better performance, we apply MCMC detection to channels with two transmit and two receive antennas. Two modulation codes are considered. However, both codes lead to inferior performance compared to that of the single transmit and two receive antenna system. Thus, even though it is theoretically possible to find better modulation codes to realize the potential gain of the two transmit antenna system, we believe that it is not a trivial task.

In other related work [9], capacity-approaching joint channel decoding and noncoherent detection strategies are developed for single antenna block fading channels with a relatively low spectral efficiency of $1 / 2$ bits/channel use with QPSK modulation. Because low-complexity noncoherent detector in [9] requires both amplitude estimation and phase quantization of the channel fading state, it is difficult to adopt large constellations with multiple amplitude levels. While a modified version of this noncoherent detector is studied in [10] to facilitate 8 QAM modulation, the detector seems to be less effective partially due to the difficulty with amplitude estimation. In comparison, the proposed MCMC detector does not require explicit amplitude estimation or phase quantization, which makes it highly adaptable to QAM modulation and can be easily generalized to MIMO channels.

The rest of the paper is organized as follows. Section II contains the system model. Section III includes a detailed description of the noncoherent MCMC detector. Simulation results of the single antenna system are presented in Section IV. In Section V, we extend MCMC detection to channels with two transmit antennas and compare their performance with that of the single transmit antenna system. Conclusions are given in Section VI.

\section{SYSTEM MODEL}

We consider a block fading channel with $M$ transmit and $N$ receive antennas. The channel is assumed to remain constant for every block of $T_{c}$ symbols, where $T_{c}$ is called the 
coherence length of the channel, and is independent between blocks. We model the channel by :

$$
\mathbf{Y}=\sqrt{\frac{\rho}{M}} \mathbf{S H}+\mathbf{W}
$$

where $\mathbf{S} \in \mathcal{C}^{T_{c} \times M}, \mathbf{Y} \in \mathcal{C}^{T_{c} \times N}, \mathbf{H} \in \mathcal{C}^{M \times N}$, and $\mathbf{W} \in \mathcal{C}^{T_{c} \times N}$, represent the transmitted signal matrix, the received signal matrix, the channel fading matrix, and the noise matrix, respectively. We assume that the entries of $\mathbf{H}$ are independently and identically distributed (i.i.d.) random variables with complex Gaussian distribution $\mathcal{C N}(0,1)$. The entries of the noise matrix are also i.i.d. with distribution $\mathcal{C N}(0,1)$. Assume that the average power of the transmitted signal $\mathbf{S}$ is normalized to be $1: E\left[\operatorname{tr}\left(\mathbf{S S}^{\dagger}\right] / M=1\right.$. The constant $\rho$ represents the signal to noise power ratio at each receive antenna. Given the transmitted signal $\mathbf{S}$, the conditional probability density function (pdf) of the received signal is [4]:

$$
p(\mathbf{Y} \mid \mathbf{S})=\frac{\exp \left[-\operatorname{tr}\left(I_{T_{c}}+(\rho / M) \mathbf{S S}^{\dagger}\right)^{-1} \mathbf{Y} \mathbf{Y}^{\dagger}\right]}{\pi^{T_{c} N} \operatorname{det}^{N}\left(I_{T_{c}}+(\rho / M) \mathbf{S S}^{\dagger}\right)}
$$

Figure 1 shows a block diagram of the system. The channel

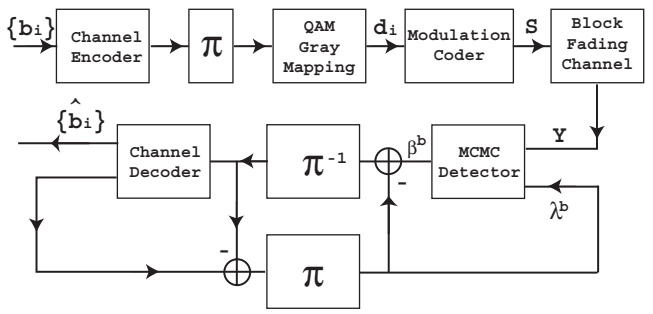

Fig. 1. A schematic block diagram of the MIMO system.

encoder encodes a sequence of information bits $\mathbf{b}$ into a sequence of coded bits. The resulting coded sequence is interleaved by a random permutation indicated by $\pi$ and mapped to a sequence of QAM symbols $\mathbf{d}$ using Gray mapping. The sequence $\mathbf{d}$ is then passed to the modulation coder and the output is sent through the block fading channel. At the receiver end, for each block of $N \cdot T_{c}$ received signal samples, the noncoherent MCMC detector computes a posteriori probabilities (APPs) of input QAM symbols. Subsequently, these symbol APPs are used to compute bit-wise APPs. The "extrinsic" part of these bit-wise APPs is then de-interleaved and passed to the channel decoder. The channel decoder performs one or more decoding iterations and generates bit-wise extrinsic information. Assuming that the bits which constitute a QAM symbol are statistically independent, the interleaved bit-wise extrinsic information $\left\{\lambda^{b}\right\}$ is fed back to the block noncoherent detector, which updates the prior symbol probabilities $\lambda^{s}$. For the next iteration, the detector computes symbol APPs using $\lambda^{s}$. In this manner, the noncoherent detection and channel decoding proceed iteratively. After a fixed number of iterations, decisions are made at the output of the channel decoder to generate the decoded bits $\hat{b}$.

\section{NONCOHERENT DETECTOR BASED ON MCMC / GibBS} SAMPLER

In this section, we explain how the noncoherent detector decodes the modulation code and generates extrinsic information regarding the transmitted bits based on prior information from the channel decoder. For simplicity, we consider the single transmit and receive antenna case with $M=N=1$. The modulation code we choose maps a block of $\left(T_{c}-1\right)$ QAM symbols to a block of $T_{c}$ QAM symbols by inserting a reference symbol $c_{0}$ (from the same QAM constellation ) in the front of each input block: $\left(s_{1}, \cdots, s_{T_{c}-1}\right) \rightarrow \mathbf{S}=$ $\left(s_{0}=c_{0}, s_{1}, \cdots, s_{T_{c}-1}\right)$, where each $s_{i}, i=1, \cdots, T_{c}-1$ is an arbitrary 16 QAM symbol. Note that the size of this modulation code is $16^{T_{c}-1}$ which grows exponentially with respect to $T_{c}$. When $T_{c}=6$, the code size equals $2^{20}$. Such a big size renders it infeasible to perform optimum detection. Hence, we must develop low-complexity detectors to make the iterative system functional.

The noncoherent detector we study here is based on the coherent MCMC detector proposed in [5], [6], [8]. For completeness of the paper, we include a detailed description of the modified noncoherent detector. The MCMC detector operates in two steps. In the first step, it adopts a statistical approach to identify a small subset of "important vectors" in the set of all possible transmitted vectors $\mathbf{S}$. This is done by running one or multiple Markov Chains (Gibbs sampler) based on the received signal samples. In the second step, the detector computes the output probabilities by averaging over only the subset of vectors found in step 1 , which greatly reduces the computational complexity.

Given each block of received samples $\mathbf{Y}=$ $\left\{y_{0}, \quad y_{1}, \cdots, y_{T_{c}-1}\right\}$ and the symbol priors $\lambda^{s}$ from the channel decoder, the Gibbs sampler operates as follows: Gibbs sampler:

$n=0$; Generate the initial $\mathbf{S}^{(0)}$ vector using the priors $\lambda^{s}$.

$\%$ In the following for-loop sample vector $\mathbf{S}^{(n)}$ is generated $\%$ by changing/sampling one symbol at a time.

for $n=1$ to $I$

generate $s_{1}^{(n)}$ using the distribution

$P\left(s_{1}=a \mid s_{0}=c_{0}, s_{2}^{(n-1)}, s_{3}^{(n-1)}, \cdots, s_{T_{c}-1}^{(n-1)}, \mathbf{Y}, \boldsymbol{\lambda}^{s}\right)$

generate $s_{2}^{(n)}$ using the distribution

$P\left(s_{2}=a \mid s_{0}=c_{0}, s_{1}^{(n)}, s_{3}^{(n-1)}, \cdots, s_{T_{c}-1}^{(n-1)}, \mathbf{Y}, \boldsymbol{\lambda}^{s}\right)$

generate $s_{T_{c}-1}^{(n)}$ using the distribution

$P\left(s_{T_{c}-1}=a \mid s_{0}=c_{0}, s_{1}^{(n)}, s_{2}^{(n)}, \cdots, s_{T_{c}-1}^{(n)}, \mathbf{Y}, \boldsymbol{\lambda}^{s}\right)$

$\%$ end for-loop

Note that in the step of generating sample $s_{i}^{(n)}$, only the prior probability for symbol $s_{i}$ is needed. For instance,

$$
\begin{aligned}
& P\left(s_{1}=a \mid s_{0}=c_{0}, s_{2}^{(n-1)}, s_{3}^{(n-1)}, \cdots, s_{T_{c}}^{(n-1)}, \mathbf{Y}, \boldsymbol{\lambda}^{s}\right) \\
& \propto p\left(\mathbf{Y} \mid s_{0}=c_{0}, s_{1}=a, s_{2}^{(n-1)}, \cdots, s_{T_{c}}^{(n-1)}\right) \cdot \lambda^{s}\left(s_{1}=a\right),
\end{aligned}
$$

which involves only $\lambda^{s}\left(s_{1}=a\right)$. The pdf in (3) is the same as the noncoherent pdf in (2). Following the procedure above, 
the Gibbs sampler runs over all symbols $I$ times to generate a collection of vectors $\mathbf{S}^{(0)}, \cdots, \mathbf{S}^{(I)}$, which are put into a $\operatorname{set} \mathcal{C}$. In [6], it is noted that obtaining Gibbs samples from a number of independent/parallel Gibbs samplers results in more robust detectors. Here, also, we run $Q$ parallel Gibbs sampler to get more samples and they are all added (except for repetitions) to the set $\mathcal{C}$.

In step 2, we compute the output symbol probabilities based on the samples in $\mathcal{C}$. let $\mathbf{S}_{-i}=$

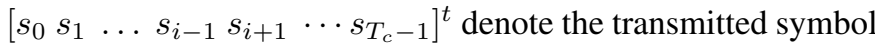
vector except for the $i$-th symbol, where $(\cdot)^{t}$ is the transpose operator. Since $s_{0}$ is a fixed reference symbol, there are a total of $16^{T_{c}-2}$ different $\mathbf{S}_{-i}$. In the following, we let $\mathbf{D}_{-i}$ represent any of such vectors. Using $\lambda^{s}$, the optimal noncoherent detector computes updated symbol-wise APPs $\beta^{s}$ as follows:

$$
\begin{aligned}
& \beta^{s}\left(s_{i}=a\right)=P\left(s_{i}=a \mid \mathbf{Y} ; \lambda^{s}\right) \\
& =\sum_{\mathbf{D}_{-i}} P\left(s_{i}=a, \mathbf{S}_{-i}=\mathbf{D}_{-i} \mid \mathbf{Y}, \lambda^{s}\right) \\
& \propto \sum_{\mathbf{D}_{-i}} p\left(\mathbf{Y} \mid s_{i}=a, \mathbf{S}_{-i}=\mathbf{D}_{-i}\right) \cdot P\left(s_{i}=a, \mathbf{S}_{-i}=\mathbf{D}_{-i} \mid \lambda^{s}\right) \\
& =\sum_{\mathbf{D}_{-i}} p\left(\mathbf{Y} \mid s_{i}=a, \mathbf{S}_{-i}=\mathbf{D}_{-i}\right) \cdot \lambda^{s}\left(s_{i}=a\right) \\
& \quad \cdot \lambda^{s}\left(\mathbf{S}_{-i}=\mathbf{D}_{-i}\right), \quad i=1, \cdots, T_{c}-1 .
\end{aligned}
$$

Note that the summation in (4) is over all possible choices of $\mathbf{D}_{-i}$, therefore the total number of terms in the summation equals $16^{T_{c}-2}$. In order to reduce complexity, the MCMC detector approximates the optimal detector (4) by

$$
\begin{aligned}
\beta^{s}\left(s_{i}=a\right) \propto & \sum_{\mathbf{D}_{-i} \in \mathcal{C}_{i}} p\left(\mathbf{Y} \mid s_{i}=a, \mathbf{S}_{-i}=\mathbf{D}_{-i}\right) \\
& \cdot \lambda^{s}\left(s_{i}=a\right) \cdot \lambda^{s}\left(\mathbf{S}_{-i}=\mathbf{D}_{-i}\right),
\end{aligned}
$$

where $\mathcal{C}_{i}$ consists of all sample vectors in $\mathcal{C}$ with their $i$-th symbol deleted. Since we require that all vectors in $\mathcal{C}_{i}$ are distinct, the size of $C_{i}$ is no more than $Q \cdot(I+1)$. Clearly, in order for (5) to be a good approximation of the optimal detector, the samples in $\mathcal{C}_{i}$ should contain a sufficient number of the "significant" terms in the summation of (4). As seen from simulations below, using the Gibbs sampler, we are able to obtain very good performances with a small number of samples.

\section{Performance of One transmit And one Receive ANTENNA SYSTEM WITH MCMC DETECTION.}

Using the MCMC noncoherent detector described in Section III, we simulate performance of the one transmit and one receive antenna system. For an overall transmission rate of $R$ bits/channel use, the rate of the channel code $R_{c}$ (when $M=N=1$ ) should satisfy

$$
R=\frac{T_{c}-1}{T_{c}} R_{c} M_{c},
$$

where $M_{c}$ is the number of bits in each transmitted symbol. Here we have $M_{c}=4$ for 16 QAM constellation. The fraction in (6) is due to the first symbol in each block of $T_{c}$ symbols being the reference symbol. Given (6), the average energy per information bit to noise ratio $E_{b} / N_{0}$ can be computed from the average signal to noise ratio $E_{s} / N_{0}$ as $\left.\frac{E_{b}}{N_{0}}\right|_{\mathrm{dB}}=\left.\frac{E_{s}}{N_{0}}\right|_{\mathrm{dB}}-$ $10 \log _{10} R$.

We consider a block fading channel with $T_{c}=6$. In order to achieve the overall transmission rates of $R=1$ and $R=1.6$, we use low-density parity check codes (LDPC) of rates $R_{c}=$ 0.3 and $R_{c}=0.48$ respectively. The code parameters (given in Table I) are found by following design methods in [11] to optimize degree sequences of the LDPC code in accordance with the MCMC detector. In our simulations, we allow the maximum number of iterations between the LDPC decoder and the MCMC detector to be 60 and we perform one iteration of MCMC detection for every 10 decoding iterations inside the LDPC decoder. The parameters of the MCMC detector is $Q=I=5$. Hence, only $Q \cdot(I+1)=30$ sample vectors are required by the MCMC detector as opposed to a total of $2^{20}$ sample vectors required for optimum detection.

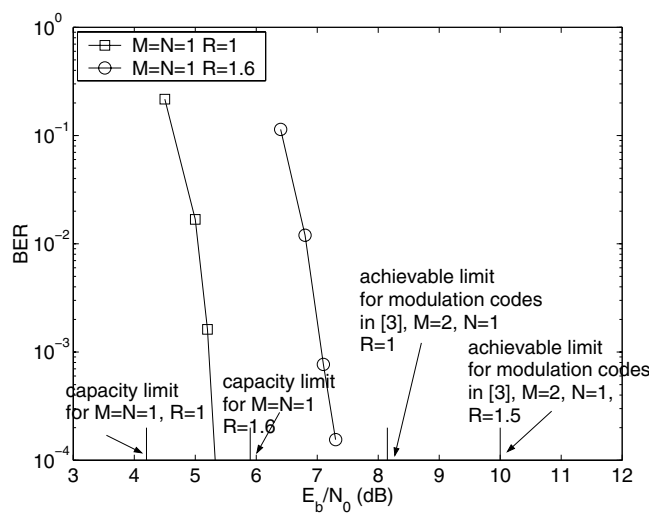

Fig. 2. Performance of the proposed system with $M=N=1$.

Based on capacity computations for the single antenna system [4], we know that with optimal input, the minimum $E_{b} / N_{0}$ required to achieve $R=1$ is about $4.2 \mathrm{~dB}$; and the minimum $E_{b} / N_{0}$ required to achieve $R=1.6$ is $5.9 \mathrm{~dB}$. Performance of our coded systems with MCMC detection and the 16 QAM modulation code are shown in Figure 2. At the bit error rate of $10^{-4}$, our system with $R=1$ is only $1.2 \mathrm{~dB}$ away from the capacity achieved by the optimal input. For the higher transmission rate of $R=1.6$, our system is $1.4 \mathrm{~dB}$ away from the capacity.

Next, we compare our results with systems that employ two transmit antennas and one receive antenna ([1]-[3]). To achieve $R=1$, two modulation codes are considered in [3]. One consists of 512 unitary space-time matrices and the other consists of 256 orthogonal space-time matrices based on the Alamouti's scheme with QPSK modulation. For the noncoherent block fading channel with $T_{c}=6$, the authors in [3] compute the mutual information achieved by these two modulation codes and determine the minimum $E_{b} / N_{0}$ required to achieve the desired transmission rates. It turns out that the 
minimum $E_{b} / N_{0}$ required are $8.15 \mathrm{~dB}$ and $8.45 \mathrm{~dB}$, respectively, for the 512 unitary codes and the 256 QPSk/Alamouti code. In comparison, as stated in the previous paragraph, it requires only $4.2 \mathrm{~dB}$ to achieve the same transmission rate for the channel with single transmit and receive antenna. Similarly, for $R=1.5$, the 8PSK/Alamouti modulation code in [3] requires a minimum $E_{b} / N_{0}$ of $10 \mathrm{~dB}$, while in the single antenna system only $5.9 \mathrm{~dB}$ is required. The achievable limits $(8.15 \mathrm{~dB}$ and $10 \mathrm{~dB})$ of the modulation codes in [3] are shown in Figure 2, and corresponding simulation curves (not shown in the figure) are about $1 \mathrm{~dB}$ away from the achievable limits. This translates into an over $4 \mathrm{~dB}$ performance gap between our single antenna system and the two transmit / single receive antenna system in [3].

We remark that such a dramatic performance difference is not because the two transmit antenna channel is intrinsically worse than the one transmit antenna channel. In fact, the capacity of the two transmit antenna channel should be no less than that of the one antenna channel because with two transmit antenna, one can always choose to allocate full power to one of the transmit antennas to realize the single antenna performance. Since it is still an open problem to compute the capacity of the two transmit antenna channel, it is not clear how much capacity gain one can expect by using two transmit antennas instead of one. However, in order to exploit the potential capacity gain promised by the two antenna channel, one should choose modulation codes that can achieve mutual information rates that are close to the capacity achieved by the optimal input. Otherwise, independent of the choices of channel codes, there will be intrinsic rate loss, as in the case of [3]. Similar unitary space-time codes (with a size of 1024 or 512) are used in [1], [2] as modulation codes. Consequently, these systems also underperform our single antenna system (by more than $6 \mathrm{~dB}$ at $R=1$ ) due to the intrinsic rate loss associated with the modulation code. Limitations of the finitesize unitary space-time codes are also discussed in [12] which shows that the mutual information achieved by these codes may only be a fraction of the optimal capacity.

\section{PERFormance of TWO TRANSMit AND TWO RECEIVE ANTENNA SYSTEMS WITH MCMC DETECTION.}

As seen from Section IV, when there is only one receive antenna, the two transmit antenna system with modulation codes such as the unitary space-time codes [3] may perform worse than our single transmit antenna system with MCMC detection. In this section, we examine the scenario with two receive antennas to see whether it is advantageous to use two transmit antennas as opposed to one.

With two transmit antennas, we consider the following two simple modulation codes. Let $\mathbf{S}=\left(\mathbf{S}_{1}, \mathbf{S}_{2}\right)$, where $\mathbf{S}_{i}$ is the transmitted signal vector from the $i$-th transmit antenna.

Modulation code 1. We do not assume $\mathbf{S}_{1}$ and $\mathbf{S}_{2}$ to be orthogonal. For example, when $T_{c}=6$, we let

$$
\left(\begin{array}{l}
\mathbf{S}_{1}^{t} \\
\mathbf{S}_{2}^{t}
\end{array}\right)=\left(\begin{array}{cccccc}
c_{0} & \times & a_{1} & a_{2} & a_{3} & a_{4} \\
\times & c_{0} & b_{1} & b_{2} & b_{3} & b_{4}
\end{array}\right) \text {. }
$$

During the last four time slots, arbitrary information symbols $a_{i}, b_{i}, i=1, \cdots, 4$ from the 16 QAM constellation are transmitted. During the first two time slots, we place reference symbols to resolve the phase ambiguity associated with unknown channel phase shift. Transmit antenna 1 sends a reference symbol $c_{0}$ during the first time slot and then sends nothing (labeled by $\times$ ) during the second time slot. Transmit antenna 2 sends nothing during the first time slot and then sends $c_{0}$ during the second time slot. This modulation code has a size of $16^{8}=2^{32}$. When it is concatenated with a LDPC code of rate $R_{c}=0.3$, we have an overall rate $R=R_{c} \cdot 32 / T_{c}=1.6 \mathrm{bits} / \mathrm{channel}$ use.

Modulation code 2. This modulation code is similar to the one proposed in [3] where $\mathbf{S}_{1}$ and $\mathbf{S}_{2}$ are made orthogonal using the Alamouti's scheme:

$$
\left(\begin{array}{c}
\mathbf{S}_{1}^{t} \\
\mathbf{S}_{2}^{t}
\end{array}\right)=\left(\begin{array}{cccccc}
c_{0} & \times & a_{1} & a_{2} & a_{3} & a_{4} \\
\times & c_{0} & -a_{2}^{\dagger} & a_{1}^{\dagger} & -a_{4}^{\dagger} & a_{3}^{\dagger}
\end{array}\right) .
$$

Note that here we use 16 QAM modulation while [3] uses smaller constellations such as QPSK or 8PSK. This modulation code has a size of $16^{4}=2^{16}$. When concatenated with a LDPC code of rate $R_{c}=0.6$, it gives an overall rate of $R=R_{c} \cdot 16 / T_{c}=1.6$ bits/channel use. Since $\mathbf{S}_{1}$ and $\mathbf{S}_{2}$ may not be orthogonal (as in modulation code 1), we need to simplify the pdf in (2) (hence avoiding the matrix inversion step) to facilitate efficient MCMC detection. First, we apply the identity $\operatorname{det}(I+C D)=\operatorname{det}(I+D C)$ to obtain

$$
\operatorname{det}\left[I_{T_{c}}+\frac{\rho}{M} \mathbf{S S}^{\dagger}\right]=\operatorname{det}\left[I_{2}+\frac{\rho}{M}\left(\begin{array}{ll}
\mathbf{S}_{1}^{\dagger} \mathbf{S}_{1} & \mathbf{S}_{1}^{\dagger} \mathbf{S}_{2} \\
\mathbf{S}_{2}^{\dagger} \mathbf{S}_{1} & \mathbf{S}_{2}^{\dagger} \mathbf{S}_{2}
\end{array}\right)\right]=\frac{\rho^{2} A}{M^{2}}
$$

with $A=\left(\frac{M}{\rho}+\left\|\mathbf{S}_{1}\right\|^{2}\right)\left(\frac{M}{\rho}+\left\|\mathbf{S}_{2}\right\|^{2}\right)-\left|\mathbf{S}_{1}^{\dagger} \mathbf{S}_{2}\right|^{2}$, and $\|$. $\|$ denotes the norm of a vector. Next, we apply the matrix inversion lemma to get

$$
\begin{aligned}
{\left[I_{T_{c}}+\frac{\rho}{M} \mathbf{S S}^{\dagger}\right]^{-1} } & =\left[\left(I_{T_{c}}+\frac{\rho}{M} \mathbf{S}_{1} \mathbf{S}_{1}^{\dagger}\right)+\frac{\rho}{M} \mathbf{S}_{2} \mathbf{S}_{2}^{\dagger}\right]^{-1} \\
& =B-B \mathbf{S}_{2} \mathbf{S}_{2}^{\dagger} B /\left[M / \rho+\mathbf{S}_{2}^{\dagger} B \mathbf{S}_{2}\right],
\end{aligned}
$$

where $B=\left[I_{T_{c}}+\frac{\rho}{M} \mathbf{S}_{1} \mathbf{S}_{1}^{\dagger}\right]^{-1}=I_{T}-\mathbf{S}_{1} \mathbf{S}_{1}^{\dagger} /\left[M / \rho+\left\|\mathbf{S}_{1}\right\|^{2}\right]$. Let the received signal matrix $\mathbf{Y}=\left(\mathbf{Y}_{1}, \mathbf{Y}_{2}\right)$, where $\mathbf{Y}_{i}$ is the received signal vector at the $i$-th receive antenna. Since the channels seen by the two receive antennas are independent, we have $p(\mathbf{Y} \mid \mathbf{S})=p\left(\mathbf{Y}_{1} \mid \mathbf{S}\right) \cdot p\left(\mathbf{Y}_{2} \mid \mathbf{S}\right)$. Substituting (7) and (8) into (2) yields

$$
\begin{aligned}
& \log \left[p\left(\mathbf{Y}_{i} \mid \mathbf{S}\right)\right]=-\operatorname{tr}\left(\mathbf{Y}_{i}^{\dagger} \mathbf{Y}_{i}\right)+\left|\mathbf{S}_{1}^{\dagger} \mathbf{Y}_{i}\right|^{2}\left(M / \rho+\left\|\mathbf{S}_{2}\right\|^{2}\right) / A+ \\
& \left|\mathbf{S}_{2}^{\dagger} \mathbf{Y}_{i}\right|^{2}\left(M / \rho+\left\|\mathbf{S}_{1}\right\|^{2}\right) / A-2 \operatorname{Re}\left[\left(\mathbf{S}_{1}^{\dagger} \mathbf{S}_{2}\right)\left(\mathbf{S}_{2}^{\dagger} \mathbf{Y}_{i}\right)\left(\mathbf{Y}_{i}^{\dagger} \mathbf{S}_{1}\right)\right] / A \\
& \quad-T_{c} N \log \pi-2 N \log (\rho / M)-\log A .
\end{aligned}
$$

In particular, if we set $\mathbf{S}_{2}=0$ and let $M=N=1$, (9) reduces to the pdf of the single antenna case. Using (9) we are able to apply the MCMC detection for the two transmit antenna case with minor modifications of the detector in Section III. For instance, the Gibbs sampler should be modified to take into account the special structures of the modulation codes. 
Simulation results are presented in Figure 3 where we examine the performance of the two transmit and two receive antenna systems with the modulation codes above. The performance of the single transmit and two receive antenna system (with the modulation code described in Section III) is also shown. To ensure the overall transmission rate of $R=1.6$, the LDPC codes of rates $R_{c}=0.3,0.6$, and 0.48 are used for systems with modulation code 1 , modulation code 2 , and the single transmit antenna system, respectively. The code parameters are found in Table I. We use the same parameters $Q=I=5$ for the MCMC detection. Interestingly, we see that the one transmit antenna system still performs the best. The two transmit antenna system with modulation code 1 , where $\mathbf{S}_{1}$ and $\mathbf{S}_{2}$ are not necessarily orthogonal, outperforms the two transmit antenna system with modulation code 2 , where $\mathbf{S}_{1}$ and $\mathbf{S}_{2}$ are orthogonal.

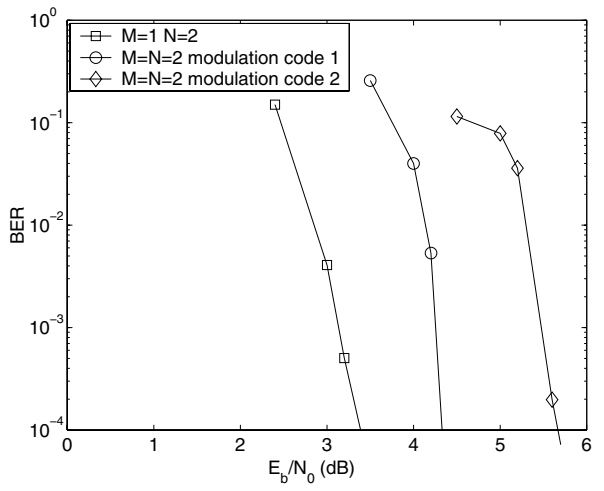

Fig. 3. Performance comparisons between the $M=1, N=2$ system and the $M=N=2$ systems with two different modulation codes.

\section{CONCLUSION}

In this paper, we study low-complexity noncoherent detectors based on the MCMC approach. Compared to other noncoherent detectors in the literature, the MCMC detector does not require explicit amplitude estimation or phase quantization. It supports large modulation codes with 16 QAM constellation and therefore can transmit at high spectral efficiency. With joint channel decoding and MCMC detection, the proposed single antenna system achieves within 1.2-1.4 dB of the channel capacity at the transmission rates of 1 and 1.6 bits/channel use. These results significantly outperform the two transmit antenna / one receive antenna systems that employ either unitary space-time code or orthogonal space-time codes as modulation codes ([1]-[3]). An interesting observation is that even with two receive antennas, the single transmit antenna system still outperforms the two transmit antenna systems for the modulation codes considered in the paper. The inferior performance of the two transmit antenna system is partly due to the use of modulation codes (such as the small size unitary space-time codes) whose achievable mutual information rates are well below the channel capacity. Hence, in order to exploit the potential gain of transmit diversity, it is crucial to develop new modulation codes that can strike a good balance between the achievable rates and the decoding complexity. We believe that the effectiveness of the MCMC detection methods will prove to be instrumental to realize such capacity gain.

TABLE I

LDPC CODE PARAMETERS

\begin{tabular}{|c|c|}
\hline $1 / 1$ & $\begin{array}{l}R_{c}=0.3 \quad \mathrm{~L}=28810 \quad d_{c}=5 \quad d_{v}=[1,3,5,7,8,27,39] \\
u_{v}=[0.59,0.266,0.03,0.098,0.005,0.01]\end{array}$ \\
\hline $1 / 1$ & $\begin{array}{l}R_{c}=0.48 \quad \mathrm{~L}=18000 \quad d_{c}=8 \quad d_{v}=[2,3,7,8,33,34] \\
u_{v}=[0.524,0.2825,0.1073,0.056,0.0132,0.0186]\end{array}$ \\
\hline $1 / 2$ & 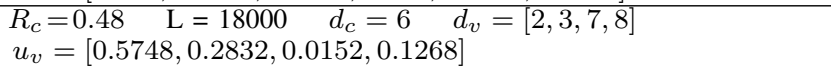 \\
\hline $2 / 2$ & $\begin{array}{l}R_{c}=0.3 \quad \mathrm{~L}=28810 \quad d_{c}=5 \quad d_{v}=[2,3,7,8,22,23,69,70] \\
u_{v}=[0.6319,0.2528,0.0011,0.0983,0.0019,0.007,0.0053,0.0017]\end{array}$ \\
\hline $2 / 2$ & $\begin{array}{llrl}R_{c}=0.6 & \mathrm{~L}=14405 & d_{c}=7 & d_{v}=[2,3,5] \\
u_{v}=[0.587,0.2194,0.1935] & \end{array}$ \\
\hline
\end{tabular}

In Table I, the first column is $M / N$ which denotes $\mathrm{M}$ transmit and $\mathrm{N}$ receive antenna, $L$ is code length, $d_{c}$ is the degree of check node, $d_{v}$ is the degree sequences of variable nodes, $u_{v}(i)$ is the fraction of variables nodes that has degree $d_{v}(i)$. The number of information bits in each LDPC code is approximately the same (either 8640 or 8643 to ensure that the code length is an integer).

\section{REFERENCES}

[1] I. Bahceci and T. M. Duman, "Trellis-coded unitary space-time modulation," IEEE Trans. on Wireless Commun., vol. 3, pp. 139-157, Nov. 2004.

[2] I. Bahceci and T. M. Duman, "Combined turbo coding and unitary spacetime modulation," IEEE Trans. on Commun., vol. 50, pp. 1244-1249, Aug. 2002.

[3] A. Krishnamoorthy and A. Anastasopoulos, "Pilot symbol assisted schemes: a better alternative to systematic unitary constellations for the non-coherent fast fading channel," Proc. of Vehicular Technology Conference (VTC), pp. 939-943, May 2004.

[4] T. Marzetta and B. Hochwald, "Capacity of a mobile multiple-antenna communication link in Rayleigh flat fading," IEEE Trans. Inform. Theory, vol. 45, pp. 139-157, Jan. 1999.

[5] H. Zhu, Z. Shi, and B. Farhang-Boroujeny, "MIMO detection using Markov chain Monte Carlo techniques for near capacity performance," IEEE ICASSP 2005, Accepted.

[6] B. Farhang-Boroujeny, H. Zhu, and Z. Shi, "Monte Carlo Markov chain techniques for CDMA and MIMO communication systems," submitted to IEEE Trans. Signal Processing.

[7] H. Zhu, B. Farhang-Beroujeny, and R. R. Chen, "On performance of sphere decoding and Markov chain Monte Carlo methods," to appear IEEE Signal Processing Letters, Oct. 2005.

[8] Z. Shi, H. Zhu, and B. Farhang-Boroujeny, "Markov chain monte carlo techniques in iterative detectors," IEEE Globecom 2004, Dallas, Nov. 29 - Dec. 22004.

[9] R. R. Chen, R. Koetter, U. Madhow, and D. Agrawal, "Joint noncoherent demodulation and decoding for the block fading channel: a practical framework for approaching shannon capacity," IEEE Trans. Commun., vol. 51, pp. 1676-1689, Oct. 2003.

[10] N. Jacobsen and U. Madhow, "Reduced-complexity noncoherent communication with differential QAM and iterative receiver processing," Proc. of the 2003 Conf. on Information Sciences and Systems (CISS 2003), Mar. 2003.

[11] S. ten Brink, G. Kramer, and A. Ashikhmin, "Design of low-density parity-check codes for modulation and detection," IEEE Trans. on Commun., vol. 52, pp. 670-678, Apr. 2004.

[12] B. M. Hochwald, T. L. Marzetta, T. J. Richardson, W. Sweldens, and R. Urbanke, "Systematic design of unitary space-time constellations," IEEE Trans. Inform. Theory, vol. 46, pp. 1962-1973, Sep. 2000. 